\title{
SALUD SEXUAL Y CALIDAD DE VIDA EN PERSONAS CON TRAUMATISMO CRANEOENCEFÁLICO MODERADO (TCEM)
}

\author{
Esther Sánchez Raja \\ Presidenta de la Asociación Nacional de Salud Sexual y Discapacidad (ANSSYD) \\ esanchezra@gmail.com \\ María Honrubia Pérez \\ Vicepresidenta de la Asociación Nacional de Salud Sexual y Discapacidad (ANSSYD) \\ mhonrubia@ub.edu
}

Fecha de Recepción: 25 Febrero 2018

Fecha de Admisión: 10 Abril 2018

\section{RESUMEN}

Los traumatismos craneoencefálicos (TCE) afectan con mayor frecuencia a personas relativamente jóvenes y constituyen una causa importante de minusvalía neurológica persistente con diferentes grados de incapacidad. El objetivo del estudio fue conocer el estado de Salud Sexual de las personas con Traumatismo Craneoencefálico Moderado (TCEM), medir su Calidad de Vida (CV) y explorar desde la perspectiva de las personas los cambios generados en su vida a consecuencia de la lesión. Participaron 184 pacientes con TCEM. Los resultados evidenciaron alteraciones en las actividades de la vida diaria, en el ámbito de relación personal, alteraciones conductuales y emocionales, cambios en la propia vida y en la familia. En cuanto a las alteraciones sexuales surgidas posteriores al TCEM, se detectan problemas sexuales persistentes y disminución de la frecuencia de relaciones coitales .Los problemas con mayor prevalencia en los hombres son la eyaculación precoz y disfunción eréctil y en las mujeres los de sequedad vaginal, la falta de excitación, de orgasmo y la dispareunia. De forma general, los cambios cognitivos, físicos, emocionales y comportamentales que ocurren tras sufrir un TCEM tienen una repercusión directa sobre la sexualidad

Palabras clave: traumatismo craneoencefálico; salud sexual; disfunciones sexuales; discapacidad; calidad de vida

\section{ABSTRACT \\ Sexual health and quality of life in people with moderate brain injury trauma (MBIT). \\ Traumatic brain injuries (TBI) affect relatively young people more frequently and are an impor- tant cause of persistent neurological disability with different degrees of disability. The objective of the study was to know the state of Sexual Health of people with Moderate Traumatic Brain Injury (MTBI), measure their Quality of Life (QL) and explore from the perspective of people the changes}


generated in their lives as a result of the injury. A total of 184 patients with MTBI participated. The results evidenced alterations in the activities of the daily life, in the scope of personal relation, behavioural and emotional alterations, changes in the own life and in the family. As for the sexual alterations arising after the MTBI, persistent sexual problems are detected and the frequency of coital relationships decreases. The problems with greater prevalence in men are premature ejaculation and erectile dysfunction and in women those with vaginal dryness, the lack of excitement, orgasm and dyspareunia. In general, the cognitive, physical, emotional and behavioural changes that occur after suffering a MTBI have a direct impact on sexuality.

Keywords: traumatic brain injury; sexual health; sexual dysfunction; disability; quality of life

\section{ANTECEDENTES}

Se denomina daño cerebral sobrevenido o adquirido (DCA), a cualquier lesión repentina en el cerebro, posterior al momento del nacimiento, ocasionado por causas mecánicas internas 0 externas (Campbell M, 2000). El daño cerebral adquirido por causas mecánicas externas es de origen traumático, por lo que suele identificarse como «Traumatismo Craneoencefálico» (TCE). El TCE se caracteriza por una serie de cambios que se presentan en una persona cuando recibe un impacto en la cabeza, comprende diversos cuadros clínicos, que pueden ir desde la conmoción cerebral hasta las lesiones más severas y complejas que pueden ocasionar la muerte. Shiffman et al, (2003),definen el TCE considerando dos situaciones: la primera, como una lesión del cuero cabelludo por contusión, lesión penetrante o por fuerza de aceleración o deceleración, que presenta al menos alguno de estos signos: alteración de conciencia, amnesia por traumatismo cerebral, cambios neurológicos, cambios neuropsicológicos, una fractura craneal o alguna lesión atribuida al traumatismo; la segunda, como aquella situación o suceso de muerte resultado de un traumatismo con lesión cerebral.

EI TCE se conoce también como la «epidemia silenciosa de nuestro tiempo», representando un grave problema de salud pública en el mundo desarrollado, constituye, con frecuencia, la cuarta causa de mortalidad tras la enfermedad cardíaca, el cáncer y el ictus, y pese a que el TCEM no suele ocasionar la muerte, sí que suele ir acompañado de una serie de secuelas que se prolongarán durante toda la vida, afectando, en muchos casos, a la calidad de vida de estos pacientes, lo que conlleva años potenciales de vida perdidos Ostabal y Sanz , (1995).

Según la Organización Mundial de la Salud (OMS 2004), el mecanismo de lesión más frecuente en los países industrializados es el accidente de tráfico, que supone la causa más importante de muerte entre la población menor de 45 años. Murillo, Gilli y Muñoz (1996) aseguran que este tipo de lesiones, contribuye al fallecimiento en el $45 \%$ de los casos con politraumatismo y es una de las principales causas de incapacidad, generando un elevado coste sanitario, social y económico para la sociedad.

También se pueden producir las lesiones por caída, por accidentes laborales, deportivos y otras causas. Tagliaferri, Compagnone, Korsic, Servadei y Kraus (2006) afirman que en los niños la causa más frecuente es por caída. Estudios realizados por Basso, Previgliano, Duarte y Ferrari (2001); Bruns, y Hauser (2003) revelan que la incidencia general de los TCE varía según sea el país, el diseño del estudio, las diferentes definiciones, las diferencias demográficas y el distinto mecanismo que produce el trauma. Es una de las enfermedades más graves de la época actual y su prevención se debe plantear con los criterios epidemiológicos propios. En España, según datos proporcionados por el Hospital de Neurorehabilitación Institut Guttman de Badalona, (2012), se produjeron entre 175 y 200 nuevos casos de TCE por cada 100.000 habitantes y año, lo que significa alrededor de personas por año padeciendo lesiones traumáticas cerebrales, siendo las lesiones provocadas por Ios TCEM responsables de 5.000 muertes anuales entre las personas de 5 a 29 años, como refleja 
Vaquero, (2007) en su obra Aspectos generales de los traumatismos craneoencefálicos :Daño cerebral postraumático. Las secuelas derivadas de estas lesiones son muy complejas presentando problemas en diversas áreas (físicas, sensoriales, cognitivas y conductuales), con las consecuentes repercusiones sobre la vida de los pacientes a todos los niveles, (actividades de la vida diaria, laboral, social, económica...) tanto para los afectados como para sus familiares, siendo éstas responsables del $40 \%$ de las nuevas grandes minusvalías.

\section{OBJETIVOS DE LA INVESTIGACIÓN}

10 fase: Estudio descriptivo transversal Objetivo General

Conocer el impacto del TCEM en la calidad de vida y en la función sexual de las personas afectadas

\section{Objetivos Específicos}

Describir las características sociodemográficas

Detectar las alteraciones sexuales existentes en las personas que han sufrido un TCEM

Medir la CV en personas con TCEM

2ㅜ Fase: Estudio cualitativo descriptivo desde la perspectiva metodológica de la etnografía enfocada.

Objetivo General

Describir la experiencia de vivir con un TCEM y sus consecuencias en la Salud Sexual y en la CV Objetivos Específicos

Explorar desde la perspectiva de las personas afectadas los cambios generados en su salud sexual y calidad de vida como consecuencia del TCEM.

Averiguar si en el proceso de rehabilitación han recibido algún tipo de asesoramiento o educación sexual sobre los posibles cambios que se pueden generar como consecuencia del TCEM.

Visualizar desde la perspectiva de las personas afectadas las repercusiones del TCE en sus relaciones interpersonales.

Generar conocimiento fundamentado en referencia a la repercusión que tienen las alteraciones sexuales en la CV

\section{SUJETOS DE ESTUDIO.}

Primera fase: La muestra final del estudio fue de 184 personas, 137 varones $(74,5 \%)$ y 47 mujeres (25,5\%), pertenecientes a las 19 Asociaciones participantes.

Segunda fase: 25 personas con TCEM que están inscritos en la Asociación de Daño cerebral ATENEO Castellón.

Se configura el grupo focal con criterios preestablecidos para lograr la suficiencia del dato determinado por Fossey et al, (2002) analizando todas las posibilidades de fuentes de información tanto en tipo de personas como de datos.

Criterios de inclusión: Personas entre 19 y 60 años que no presentasen una afectación cognitiva que les impidiera comprender y rellenar los cuestionarios y participar en los grupos focales, la inexistencia de otras enfermedades de carácter crónico degenerativo asociadas al TCEM y que hubiera pasado como mínimo un año de haber sufrido la lesión cerebral

\section{METODOLOGÍA E INSTRUMENTOS}

Diseño mixto fundamentado en el concepto de la triangulación de datos:

Primera fase del estudio: Estudio cuantitativo, descriptivo, transversal.

Para medir la CV se utilizó el cuestionario genérico SF36 por estar validado en España para este tipo de pacientes, Villarreal (2012), Campbell (2000), Shiffman et al (2003). 
Autores como, Ostabal y Sanz (1995), World Health Organization (2004), Murillo, Gilli y Muñoz, (1996), Tagliaferri et al (2006) recomiendan esperar al menos un año para medir la CV de estos pacientes y utilizar el cuestionario SF36 debido a su calidad psicométrica. Las 36 preguntas del cuestionario miden 8 escalas de salud. Estas 8 escalas, a su vez, son agregadas en dos medidas de resumen; un componente de Salud Física (CSF) y un segundo componente de Salud Mental (CSM).

Para conocer el impacto del TCEM en la función sexual de las personas afectadas se utilizan los cuestionarios EVAS- $\mathrm{H}$ y EVAS- $\mathrm{M}$ basados en el cuestionario FSFI elaborado y validado por Fernández, Lacalle y García (2009), La Calle, García, De la Fuente y Fernández (2008), La Calle et al (2013), Sánchez y Honrubia (2013), se trata de un cuestionario breve, sencillo auto administrado y fácil de aplicar.

Para la recogida de información se contactó con FEDACE, se solicitó participación y colaboración para coordinar el reparto de los cuestionarios por las diferentes asociaciones, seleccionar las personas que cumplieran los criterios de inclusión y hacerse cargo de la recogida de los mismos una vez estuvieran cumplimentados. Los datos se recogieron entre enero y mayo de 2014.

Para el análisis de los test SF-36, EVAS-H y EVAS-M se siguieron las instrucciones de estandarización del contenido y puntuación de la versión española de los test. Se realizó un análisis descriptivo de las variables y se construyeron los intervalos de confianza al 95\%. Para el estudio de la posible asociación entre variables cualitativas se utilizó la prueba ji-cuadrado o la prueba exacta de Fisher según las características de los grupos y sus tamaños muestrales.

Para el estudio de las relaciones bivariadas entre variables cuantitativas y cualitativas se utilizaron las pruebas t de student, Anova, $\mathrm{U}$ de Mann- Whitney y

Kruskall-Wallis dependiendo de las características de los grupos y tamaños muestrales. Para determinar el perfil de las personas con disfunción sexual y, en consecuencia, su relación con las diferentes variables consideradas en el estudio, se construyó un modelo de regresión logística en el que se contemplaron los posibles efectos de interacción. El análisis se realizó con el programa estadístico SPSS18.0.

Segunda fase del estudio: Se abordó con un diseño metodológico cualitativo, aplicando la Etnografía Enfocada para describir y comprender las alteraciones que se producen en la sexualidad de las personas después de sufrir un TCEM y como éstas pueden afectar su C.V.

El análisis correspondiente al estudio cualitativo se realizó de forma manual a partir de la trascripciones de los discursos de los grupos focales, de los relatos etnográficos y del vaciado del trabajo de campo. Para el análisis de datos se ha realizado un análisis de contenido temático siguiendo las etapas propuestas por Miles y Huberman(1994). La observación se ha realizado en la Asociación de pacientes y en el grupo focal limitándose a la observación de las relaciones interpersonales y en el posicionamiento personal ante el hecho de hablar de su experiencia en el grupo focal.

\section{ASPECTOS ÉTICOS}

Este proyecto se llevó a cabo teniendo en cuenta la legislación vigente y acatando las normas éticas de la Declaración de Helsinki y de Buena Práctica Clínica y de Laboratorio. El tratamiento, comunicación y la cesión de los datos de carácter personal de todos los sujetos participantes se ajusta a la Ley Orgánica española 15/1999 de 13 de diciembre de protección de datos de carácter personal, garantizando la confidencialidad a nivel de protección de la legislación vigente de nuestro país, Agencia Estatal Boletín Oficial del Estado. Ley Orgánica 15/1999, (2006).

\section{RESULTADOS}

El Análisis de las ocho dimensiones de cuestionario SF36 ninguna dimensión se sobrepasa Ios 22,86 puntos en hombres y 23,98 en mujeres. Por otra parte puede observarse que las puntua- 
ciones en hombres y mujeres fueron extremadamente similares en todas las dimensiones del cuestionario, no detectándose diferencias significativas en ninguno de los casos. El análisis comparativo de las puntuaciones de cada una de las dimensiones del cuestionario SF36 obtenidas en la muestra con las correspondientes a las puntuaciones medias de la población española de referencia evidenció diferencias muy significativas en todas y cada una de las dimensiones, tanto en hombres como en mujeres (Véase tabla 1)

Tabla 1: Comparación de las puntuaciones medias del SF-36 para España y la muestra según dimensiones y sexo

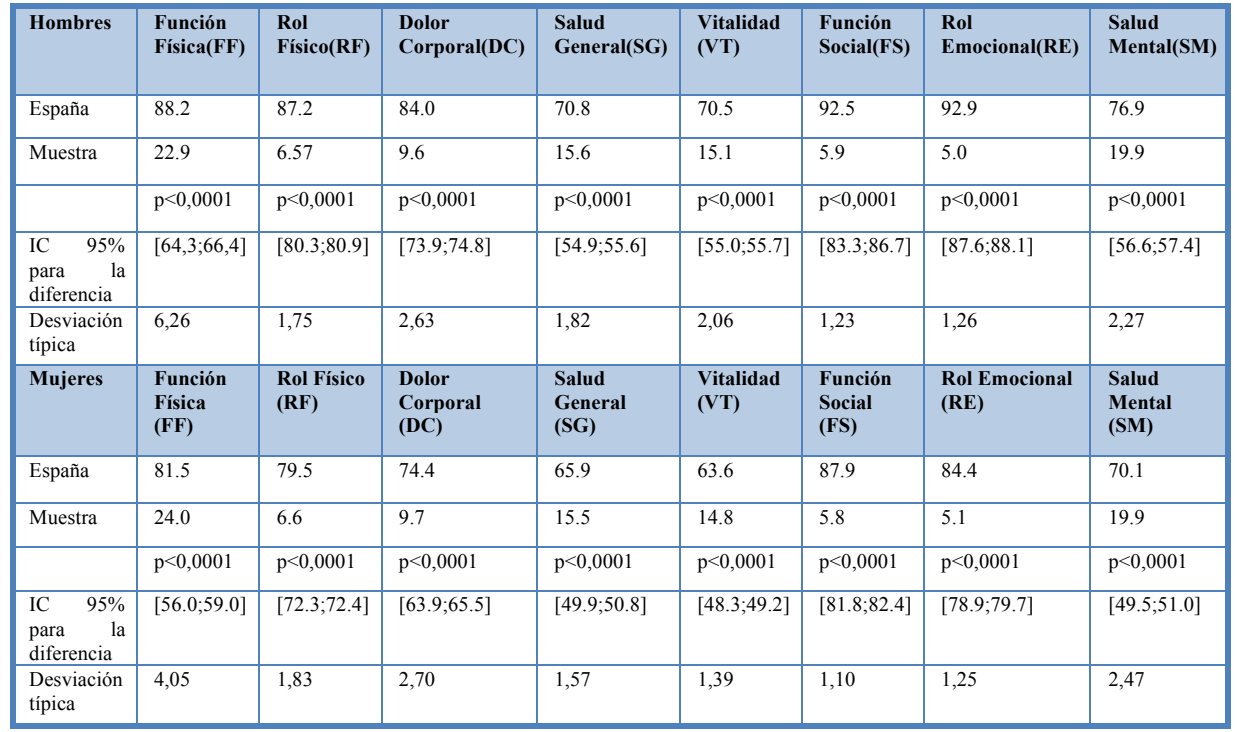

En el análisis de cada una de las preguntas de los cuestionarios EVAS-H; EVAS-M desagregadas por sexo, se observaron puntuaciones medias significativamente mayores en hombres para las dimensiones Satisfacción, Tiempo de insatisfacción, Eyaculación/Lubricación y Orgasmo.

Las puntuaciones medias fueron significativamente mayores en mujeres para las dimensiones dificultades de excitación, dolor y cercanía; mientras que en el caso del Interés sexual no se detectaron diferencias significativas.

El análisis de estas mismas dimensiones por categorías permitió comprobar que más de la mitad de la muestra $(55,7 \%)$ está muy o bastante insatisfecha con su actividad sexual, siendo este porcentaje mayor en las mujer.

En la valoración de las dimensiones sexuales por sexos, no se observaron diferencias significativas en el interés sexual entre hombres y mujeres (78,3\% respecto al $47,2 \%$ en los hombres), siendo los problemas de excitación significativamente más frecuentes en mujeres que en hombres. Además, los hombres tienen problemas de eyaculación precoz (35,2\% nunca tiene problemas) con más frecuencia que las mujeres los tienen de sequedad vaginal (66,7\% nunca tienen problemas). En cuanto al orgasmo, los hombres lo alcanzan significativamente con más frecuencia que las mujeres (el $34,7 \%$ de los hombres lo alcanza casi siempre frente al 2,2\% de las mujeres). Por su parte, el dolor es más frecuente en mujeres. Todas estas dimensiones quedan reflejadas en la tabla 2 
Tabla 2: Análisis de dimensiones sexuales por sexo

\begin{tabular}{|c|c|c|c|c|c|c|c|}
\hline \multicolumn{2}{|l|}{ SEXO } & \multirow{2}{*}{$\begin{array}{l}\text { Interés } \\
\text { sexual }\end{array}$} & \multirow{2}{*}{$\begin{array}{l}\text { Dific. } \\
\text { Excitac. }\end{array}$} & \multirow{2}{*}{$\begin{array}{l}\begin{array}{l}\text { Eyacul./ } \\
\text { Lubricac. }\end{array} \\
35,2 \%\end{array}$} & \multirow{2}{*}{$\begin{array}{l}\text { Orgasmo } \\
9,1 \%\end{array}$} & \multirow{2}{*}{\begin{tabular}{|l} 
Dolor \\
$70,6 \%$
\end{tabular}} & \multirow{2}{*}{$\begin{array}{l}\text { Cercanía/ } \\
\text { Confianza } \\
5,0 \%\end{array}$} \\
\hline Hombre & Nunca & & & & & & \\
\hline & Raramente & $8,2 \%$ & $13,7 \%$ & $23,0 \%$ & $9,1 \%$ & $22,7 \%$ & $5,0 \%$ \\
\hline & A veces & $30,3 \%$ & $11,3 \%$ & $30,3 \%$ & $26,4 \%$ & $5,0 \%$ & $10,7 \%$ \\
\hline & A menudo & $32,0 \%$ & $4,8 \%$ & $9,0 \%$ & $20,7 \%$ & $0,8 \%$ & $11,6 \%$ \\
\hline & $\begin{array}{l}\text { Casi siempre- } \\
\text { siempre }\end{array}$ & $23,0 \%$ & $4,0 \%$ & $2,5 \%$ & $34,7 \%$ & $0,8 \%$ & $10,7 \%$ \\
\hline \multirow[t]{5}{*}{ Mujer } & Nunca & $6,5 \%$ & $31,1 \%$ & $66,7 \%$ & $11,1 \%$ & $53,3 \%$ & 60,0 \\
\hline & Raramente & $4,3 \%$ & $26,7 \%$ & $28,9 \%$ & $37,8 \%$ & $31,1 \%$ & 20,0 \\
\hline & A veces & $19,6 \%$ & $33,3 \%$ & $4,4 \%$ & $22,2 \%$ & $15,6 \%$ & 11,1 \\
\hline & A menudo & $43,5 \%$ & $8,9 \%$ & & $26,7 \%$ & & 6,7 \\
\hline & $\begin{array}{l}\text { Casi siempre- } \\
\text { siempre }\end{array}$ & $26,1 \%$ & & & $2,2 \%$ & & 2,2 \\
\hline
\end{tabular}

En la tabla 3 se muestran las alteraciones sexuales posteriores al TCEM por dimensiones. Los resultados muestran, en hombres, diferencias significativas en todas las dimensiones consideradas dependiendo de la presencia o ausencia de "disfunción sexual". En el caso de las mujeres también se observan diferencias significativas en la mayoría de las dimensiones consideradas a excepción de las correspondientes a «Satisfacción» y «Cercanía y confianza» $(p=0,457$ y $p=0,294$ respectivamente).

Tabla 3: Disfunción sexual y dimensiones del cuestionario EVAS

\begin{tabular}{|c|c|c|c|c|c|c|}
\hline \multicolumn{6}{|c|}{ Rangos } & \multirow[b]{2}{*}{ Sig } \\
\hline \multicolumn{2}{|l|}{ Sexo } & \multirow{2}{*}{\begin{tabular}{l|l}
\multicolumn{2}{l}{ Punto corte } \\
&, 00
\end{tabular}} & \multirow{2}{*}{$\begin{array}{l}\mathbf{N} \\
44\end{array}$} & \multirow{2}{*}{$\begin{array}{l}\text { Rango } \\
\text { promedio }\end{array}$} & \multirow{2}{*}{$\begin{array}{ll}\begin{array}{l}\text { Suma de } \\
\text { rangos }\end{array} & \text { de } \\
3416,00 & \end{array}$} & \\
\hline \multirow[t]{18}{*}{ HOMBRE } & \multirow[t]{3}{*}{$\begin{array}{l}\text { 3. Durante las últimas } 4 \text { semanas ¿ha tenido interés en tener } \\
\text { algún tipo de actividad sexual? }\end{array}$} & & & & & \multirow{2}{*}{$p<0,001$} \\
\hline & & 1,00 & 73 & 47,77 & 3487,00 & \\
\hline & & Total & 117 & & & \multirow{4}{*}{$p<0,001$} \\
\hline & \multirow{3}{*}{$\begin{array}{l}\text { 4. En las relaciones sexuales ¿ha tenido dificultad en sentirse } \\
\text { excitado/a? }\end{array}$} &, 00 & 44 & 47,50 & 2090,00 & \\
\hline & & 1,00 & 73 & 65,93 & 4813,00 & \\
\hline & & Total & 117 & & & \\
\hline & \multirow{3}{*}{$\begin{array}{l}\text { 5. En las relaciones sexuales ¿con qué frecuencia ha sentido que } \\
\text { eyaculaba demasiado pronto/sequedad vaginal? }\end{array}$} &, 00 & 44 & 48,64 & 2140,00 & \multirow{3}{*}{$p=, 007$} \\
\hline & & 1,00 & 73 & 65,25 & 4763,00 & \\
\hline & & Total & 117 & & & \\
\hline & \multirow{3}{*}{$\begin{array}{l}\text { 6. Cuándo ha tenido relaciones sexuales con o sin penetración } \\
\text { ¿ha sentido orgasmo? }\end{array}$} &, 00 & 44 & 83,48 & 3673,00 & \multirow{3}{*}{$\mathbf{p}<0,001$} \\
\hline & & 1,00 & 73 & 44,25 & 3230,00 & \\
\hline & & Total & 117 & & & \\
\hline & \multirow[t]{3}{*}{ 1. ¿Está satisfecho/a en general con su actividad sexual? } &, 00 & 44 & 76,01 & 3344,50 & \multirow{3}{*}{$\mathbf{p}<0,001$} \\
\hline & & 1,00 & 73 & 48,75 & 3558,50 & \\
\hline & & Total & 117 & & & \\
\hline & \multirow[t]{3}{*}{$\begin{array}{l}\text { 9. Siente cercanía y confianza con su pareja en las relaciones } \\
\text { sexuales? }\end{array}$} &, 00 & 44 & 75,90 & 3339,50 & \multirow[t]{3}{*}{$\mathbf{p}<0,001$} \\
\hline & & 1,00 & 73 & 48,82 & 3563,50 & \\
\hline & & Total & 117 & & & \\
\hline
\end{tabular}




\begin{tabular}{|c|c|c|c|c|c|c|}
\hline & \multirow[t]{3}{*}{$\begin{array}{l}\text { 7. ¿Ha sentido algún tipo de dolor que le haya impedido terminar } \\
\text { su relación sexual? }\end{array}$} &, 00 & 44 & 50,55 & 2224,00 & \multirow[t]{3}{*}{$\mathrm{p}=\mathbf{0 , 0 0 9}$} \\
\hline & & 1,00 & 73 & 64,10 & 4679,00 & \\
\hline & & Total & 117 & & & \\
\hline \multirow[t]{21}{*}{ MUJER } & \multirow[t]{3}{*}{$\begin{array}{l}\text { 3. Durante las últimas } 4 \text { semanas ¿ha tenido interés en tener } \\
\text { algún tipo de actividad sexual? }\end{array}$} &, 00 & 33 & 26,15 & 863,00 & \multirow{3}{*}{$p=0,005$} \\
\hline & & 1,00 & 12 & 14,33 & 172,00 & \\
\hline & & Total & 45 & & & \\
\hline & \multirow{3}{*}{$\begin{array}{l}\text { 4. En las relaciones sexuales iha tenido dificultad en sentirse } \\
\text { excitado/a? }\end{array}$} &, 00 & 33 & 19,24 & 635,00 & \multirow{4}{*}{$p<0,001$} \\
\hline & & 1,00 & 12 & 33,33 & 400,00 & \\
\hline & & Total & 45 & & & \\
\hline & \multirow{3}{*}{$\begin{array}{l}\text { 5. En las relaciones sexuales ¿con qué frecuencia ha sentido que } \\
\text { eyaculaba demasiado pronto/sequedad vaginal? }\end{array}$} &, 00 & 33 & 20,71 & 683,50 & \\
\hline & & 1,00 & 12 & 29,29 & 351,50 & \multirow{2}{*}{$\mathrm{p}=\mathbf{0 , 0 1 9}$} \\
\hline & & Total & 45 & & & \\
\hline & \multirow[t]{3}{*}{$\begin{array}{l}\text { 6. Cuándo ha tenido relaciones sexuales con o sin penetración } \\
\text { ¿ha sentido orgasmo? }\end{array}$} &, 00 & 33 & 26,12 & 862,00 & \multirow[t]{3}{*}{$p=0,006$} \\
\hline & & 1,00 & 12 & 14,42 & 173,00 & \\
\hline & & Total & 45 & & & \\
\hline & \multirow[t]{3}{*}{ 1. ¿Está satisfecho/a en general con su actividad sexual? } &, 00 & 33 & 22,18 & 732,00 & \multirow{3}{*}{$\mathrm{p}=\mathbf{0 , 4 5 7}$} \\
\hline & & 1,00 & 12 & 25,25 & 303,00 & \\
\hline & & Total & 45 & & & \\
\hline & \multirow{3}{*}{$\begin{array}{l}\text { 9. Siente cercanía y confianza con su pareja en las relaciones } \\
\text { sexuales? }\end{array}$} &, 00 & 33 & 24,09 & 795,00 & \multirow[t]{2}{*}{$\mathrm{p}=\mathbf{0 , 2 9 4}$} \\
\hline & & 1,00 & 12 & 20,00 & 240,00 & \\
\hline & & Total & 45 & & & \\
\hline & \multirow[t]{3}{*}{$\begin{array}{l}\text { 7. ¿Ha sentido algún tipo de dolor que le haya impedido terminar } \\
\text { su relación sexual? }\end{array}$} &, 00 & 33 & 19,15 & 632,00 & \multirow{3}{*}{$\mathbf{p}<0,001$} \\
\hline & & 1,00 & 12 & 33,58 & 403,00 & \\
\hline & & Total & 45 & & & \\
\hline
\end{tabular}

En cuanto a los resultados del estudio cualitativo El proceso de categorización finalizó cuando no se encontraron nuevas informaciones que permitieran la creación de nuevas categorías, posteriormente se analizaron las categorías por separado, se realizaron comparaciones y relaciones entre las categorías y finalmente se clasificaron en seis grupos temáticos o Metacategorías (tabla 4).

Tabla 4: Categorías y temas emergentes

\begin{tabular}{|c|c|}
\hline Categorías & Temas Emergentes \\
\hline $1-2-10-16-18-20-22$ & Se rompió la vida \\
\hline $3-4-15-16-17$ & Adaptarse a las nuevas situaciones \\
\hline $9-11$ & Los amigos de verdad \\
\hline $5-6-7-8-19$ & El papel de la familia \\
\hline $12-13-14$ & Sexualidad \\
\hline $5-11-12-19-21$ & Expectativas de futuro \\
\hline
\end{tabular}

1ấSe rompió la vida: A pesar del daño cerebral adquirido todas las personas son conscientes del impacto negativo que generó en sus vidas y de la pérdida de sus expectativas de futuro.

2ªdaptarse a la nueva situación: Las personas entrevistadas reconocen el impacto vital del TCEM y la dificultad de adaptarse a la nueva situación. Algunos informantes refieren no haber conseguido acomodarse observándose actitudes de rebeldía y de culpabilización con independencia del tiempo transcurrido desde el accidente 
3느os amigos de verdad: Los resultados muestran que, como consecuencia del TCEM, se produce una pérdida de las amistades anteriores y se generan nuevas relaciones, en la mayoría de ocasiones entre el mismo grupo de afectados, en las asociaciones de pacientes con TCEM

$4^{\text {a }}$ El papel de la familia: EI TCEM no solo tiene un impacto individual en la persona que lo padece, sino que impacta a toda la familia ya que modifica las relaciones entre los miembros, los roles que cada uno ejerce, las expectativas y los planes de futuro.

\section{5ㄴa sexualidad}

La totalidad de los participantes en el estudio refieren cambios significativos en relación a su respuesta sexual después del TCEM, aunque no todos los informantes presentan los mismos cambios. Una de las fases de la sexualidad más afectada es la dimensión del deseo: hipersexualidad 0 hiposexualidad que corresponden a un aumento 0 una disminución de la libido.

La mayoría afirman que no tienen ninguna apetencia sexual siendo las mujeres las más afectadas en este aspecto. Los informantes generan un sentimiento de inseguridad y de falta de atractivo sexual, los que conviven con la familia se sienten vigilados y controlados económicamente y socialmente lo cual merma su libertad y su autonomía para satisfacer sus necesidades sexuales. Se aprecia un cambio en las formas de satisfacerse sexualmente. Mientras que en las mujeres se da con poca frecuencia la masturbación y la utilización de la asistencia sexual, estas son las prácticas más utilizadas entre los varones.

6ªxpectativas de futuro: En general, no quieren plantearse expectativas a largo plazo. Sólo un informante de sexo femenino nos hizo partícipe de su deseo de ser madre posterior al TCEM

\section{DISCUSIÓN}

Las características demográficas de la muestra estudiada, fueron similares en cuanto a edad, sexo, nivel de estudios y agente causal a los estudios publicados en España por Ladera Fernández et. Al (2008), el informe realizado por el Ministerio de Sanidad, Política Social e Igualdad (2011) y también, a los estudios publicados en Europa por Patel et. al (2005) y Tagliaferri et. al (2006)

Los resultados de estudios realizados por Dijkers (2004), Findler et al (2001), Emanuelson (2003) han mostrado que en casi todas las dimensiones analizadas, de la escala SF36 las personas con TCEM tienen una puntuación más baja que la población de referencia, estos datos coinciden con los resultados obtenidos en esta investigación, observándose que las puntuaciones en hombres y mujeres fueron extremadamente similares en todas las dimensiones del cuestionario.

En las aportaciones que proporcionan los informantes de los grupos focales, se confirma que el TCEM también afecta las relaciones familiares y sociales, los informantes vivían en entornos limitados a su círculo familiar, con frecuencia en una situación de aislamiento y de falta de apoyo social, datos que coinciden con las investigaciones realizadas por Johnson y Davis(1998).

En cuanto a los cambios en sus relaciones interpersonales, tanto el estudio cuantitativo como el cualitativo reflejan una serie de variaciones a niveles de amistad, proyección de trabajo, vida social y expectativas, experimentando por parte de éstos un sentimiento, a veces muy arraigado, de desengaño, pérdida de autoestima, de atractivo sexual.

Los resultados ponen de manifiesto que los cambios en el funcionamiento sexual son comunes después de sufrir un TCEM. La respuesta y la expresión sexual, se ven alteradas por las secuelas físicas, neuropsicológicas, emocionales y conductuales. Los datos cuantitativos refieren variaciones sexuales posteriores al TCEM en todos los niveles anteriormente mencionados, comprobando que más de la mitad de la muestra $(55,7 \%)$ está muy o bastante insatisfecha con su actividad sexual, siendo este porcentaje mayor en las mujeres (78,3\% respecto al $47,2 \%$ en los hombres). También se detectan diferencias significativas en la prevalencia de disfunción sexual según el sexo siendo 
más frecuente en varones ( $62,3 \%$ frente a un $26,6 \%$ en mujeres). Los problemas con mayor prevalencia en los hombres son los de eyaculación precoz y disfunción eréctil y en las mujeres los de sequedad vaginal y problemas de excitación.

Estos datos que coinciden con investigaciones realizadas por Kosteljanetz et al(1981), Clinchot et al (1998), Hibbard et al (2009), Glenn et al (2001), Oddy(2001), Borgaro et al(2003), Ponsford (2003), Howes et al (2005), Parcell (2006), Brent E. et al(2010) Sanders(2012), Sánchez (2015), y se fortalecen por las informaciones aportadas por los/las asistentes a los grupos de discusión de la investigación cualitativa.

La mayoría de los varones que no tienen pareja estable utilizan los servicios de trabajadoras sexuales para satisfacer sus necesidades, lo que no ocurre en el caso de las mujeres de nuestro estudio que no solicitan en ningún caso sexo de pago. La masturbación es una de las prácticas sexuales más utilizadas por los varones tras sufrir la lesión cerebral, pero tampoco esta actividad es refrendada por la mayoría de las mujeres. Todos los informantes, hombres y mujeres, mencionan una carencia de Educación afectivo sexual posterior al TCEM datos que coinciden con el estudio realizado por Bélanger (2009).

No se pudo establecer una relación entre los resultados obtenidos en el SF36 (cuestionario de CV) y el EVAS-M y EVAS-H (alteraciones sexuales) en el estudio cuantitativo; Sin embargo, debe tenerse en cuenta que esta ausencia de relación podría ser explicada por la escasa variabilidad observada tanto en hombres como mujeres en las puntuaciones del SF36. Por el contrario, en la investigación cualitativa los informantes interpretan el TCEM como un acontecimiento que alteró significativamente su vida sexual e influyó negativamente en su calidad de vida. En esta investigación no se han encontrado incongruencias entre los datos obtenidos cuantitativa y cualitativamente, por lo que podemos decir que la triangulación nos ha proporcionado una visión holística, múltiple y sumamente enriquecedora del tema.

\section{CONCLUSIONES}

El presente trabajo de investigación, a tenor de las búsquedas bibliográficas realizadas para poder fundamentar los resultados presentados, es el primero tan amplio, que se realiza a nivel nacional en todo el Estado para valorar la CV y las características de la sexualidad en las personas que han sufrido un TCEM.

Los resultados de la investigación refieren que las características sociodemográficas de la muestra constatan que las personas con TCEM son con mayor frecuencia varones jóvenes. Los cambios que se presentan después de sufrir un TCEM, repercuten directamente sobre la familia, ya que los cuidados que precisan estos pacientes son proporcionados mayoritariamente en el ámbito familiar, provocando que gran parte de los afectados se encuentren con frecuencia, en una situación de aislamiento, de falta de apoyo social, con pocos amigos y con falta evidente de contactos sociales.

La mayoría de personas con TCEM han de depender de la supervisión de un familiar por lo que la capacidad de autonomía de los afectados queda reducida y el empoderamiento como personas con capacidad de decisión limitada.

Los cambios en el funcionamiento sexual son comunes después de sufrir un TCEM. La respuesta y la expresión sexual queda alterada por las secuelas físicas, neuropsicológicas, emocionales y conductuales, disminuyen o desaparecen los encuentros eróticos debidos a diferentes factores: falta de autoestima, que provoca pensamientos negativos de miedo al fracaso y al rechazo; estados de depresión y ansiedad por las limitaciones y cambios; falta de deseo sexual producido por la medicación; sobreprotección familiar; falta de intimidad; dependencia y disminución de la vida social. 


\section{SALUD SEXUAL Y CALIDAD DE VIDA EN PERSONAS CON TRAUMATISMO CRANEOENCEFÁLICO MODERADO (TCEM)}

Los problemas de excitación, orgasmo y dispareunia son significativamente más frecuentes en mujeres que en hombres.

La masturbación es una práctica sexual comúnmente utilizada por los varones con TCEM mientras que las mujeres la practican con menor frecuencia.

Los hombres que no tienen pareja estable y viven fuera del entorno familiar, contratan más los servicios sexuales de pago que los que viven en el entorno familiar. Las mujeres no lo hacen con independencia del tipo de convivencia

La educación sexual es un pilar fundamental en la inserción social de las personas con TCEM, todos los informantes, mencionan una carencia de Educación afectivo sexual posterior al TCEM y los datos reflejan la importancia de concienciar y educar a la familia para que pueda facilitar la intimidad y la posibilidad de relaciones coitales puesto que se ha puesto en evidencia que las personas que conviven en el entorno familiar, tienen menos posibilidades de satisfacer sus necesidades sexuales que los que viven solos o en pisos compartidos alejados del entorno familiar.

\section{BIBLIOGRAFÍA}

Agencia Estatal Boletín Oficial del Estado. Ley Orgánica 15/1999, de 13 de diciembre de Protección de Datos de Carácter Personal. BOE 298. [Internet][Citada

10 marzo 2018]. Accesible en https://boe.es/boe/dias/1999/12/14/pdfs/A43088- 43099.pdf

Basso, A., Previgliano, I., Duarte, J.M., Ferrari, N. (2001). Advances in management of neurosurgical trauma in different continents World J Surg; 25(9):1174-1178.

Bélanger, D. (2009). Traumatic brain injury and sexual rehabilitation. Sexologies. 18: 83-85.

Borgaro, S., Prigatano, G., Kwásnica, C., Rexer, J. (2003). Cognitive and affective squealed in complicated and uncomplicated mild traumatic brain injury.Brain Inj.; 17:189-198.

Brent, E.M, De Witt, D.S. (2010). Diario de neurotraumatológica.; 27(8): 1529- 1540.

Bruns, J. Hauser, W.A. (2003). The epidemiology of traumatic brain injury: a review. Epilepsy. 44(s10):2-10.

Campbell, M. (2000). Rehabilitation for Traumatic Brain Injury. Physical.Therapy. Practice in Context United Kingdom: Churchill Livingstone; 17-44.

Clinchot, D.M., Bogner, J., Mysiw, W.J, Fugate, L., Corrigan, J. (1998). Defining sleep disturbance after brain injury. Am J Phys Med Rehabil; 77:291-295.

Dijkers, M.P. (2004). Quality of life after traumatic brain injury: a review of research approaches and findings. Archives of Physical Medicine and Rehabilitation; 85(s2):21-35.

Emanuelson, I., et al. (2003). Quality of life and post-concussion symptoms in adults after mild traumatic brain injury: a population-based study in western Sweden. Acta Neurológica Scandinavica; 108(5): 332-338.

Findler, M., Cantor, J., Haddad, L., Gordon, W., Ashman, T. (2001). The reliability and validity of the SF-36 health survey questionnaire for use with individuals with traumatic brain injury. Brain Injury; 15 (8): 715-723

Fossey, et al (2002). Understanding and evaluating qualitative research. Australian and New Zealand Journal of Psychiatry; 36 (6):717-732

Glenn, M.B., O'Neil-Pirozzi, T., Goldstein, R., Burke, D., Jacob, L. (2001). Depression amongst outpatients with traumatic brain injury. Brain Inj; 15: 811-818.

Hibbard, M.R., Gordon, W.A., Flanagan, S., Haddan, L., Labinsky, E. (2000). Sexual dysfunction after traumatic brain injury. Neuro Rehabilitation; 15:107- 120.

Hospital de Neurorehabilitación Institut Guttman. 2012. Informe anual. [Internet] [Citado 3 Marzo 2014]. Disponible en: http://www.guttmann.com/es. 
Howes, H., Edwards, S., Benton, D. (2005). Male body image following acquired braininjury. Brain Inj; 19:135-47.

Johnson, K., Davis, P.K. (1998). A supported relationships intervention to increase the social integration of persons with traumatic brain injuries. Behav. Modif; 22:502-528

Kosteljanetz, M., Jensen, T., Norgard, B., Lunde, I., Jensen, P., Johnsen, S. (1981). Sexual and hypothalamic dysfunction in the post-concussional syndrome. Acta Neurológica Scandinavica; 63:169-80.

La Calle, M. P, García, G.J., De la Fuente, S.M. (2009). Escala de valoración de la actividad sexual en la mujer (EVAS_M). Validación a través de entrevista con experto. Sexología integral; 6(4):160166.

La Calle, M. P., García, G.J., De la Fuente, S.M., Fernández, A. (2008). Escala de valoración de la actividad sexual en la mujer (EVAS_M). Construcción y análisis preliminares. Sexología integral; 5(3):114-116.

La Calle, M. P., Fernández, A.I., Serra, D.I., Liñán, B.R., García, C.M. (2013). Prevalencia de disfunción sexual masculina según el cuestionario EVAS-H. In: García Rojas, A.D.; Cabello Santamaría, F. Actualizaciones en sexología clínica y educativa. Huelva: Servicio. Publicaciones universidad de Huelva; p. 321-322.

Ladera, F.V., Perea, B.M., Morales, R.F. (2002). Rendimientos mnémicos en traumatismo craneoencefálico moderado al año. Psicothema. Coden Psoteg; 14: 71-76.

Miles, M., Huberman, M. (1994). Qualitative data analysis: An expanded sourcebook.NewburyParck, CA: Sage;

Ministerio de Sanidad, Política Social e Igualdad. Grupo de Trabajo de la Sociedad Española de Epidemiología sobre la Medida del Impacto en la Salud de las Lesiones por Traumatismos. Lesiones Medulares Traumáticas y Traumatismos Craneoencefálicos en España, 2000-2008. (2008). [Internet] [Citada 10 marzo 2018].Disponible:http://www.aspb.cat/quefem/docs /Lesiones_Medulares_Espania 2000_08.

Murillo, F., Gilli, M., Muñoz, M.A. (1996). Epidemiología del traumatismo craneoencefálico. En: Net, A., Marzoruecos-Sant, L. Traumatismo craneoencefálico grave. Ed. Springer-Verlag. Barcelona: Ibérica; 1-9.

Oddy, M. (2001). Sexual relationships following brain injury. Sex Relationsh Ther; 16: 247-259.

Ostabal, M.I., Sanz, S.C. (1995). Epidemiología, complicaciones y secuelas en el TCE. Rev. Esp. Neurol ;( 10): 20-25.

Patel, H.C., et al (2003). On behalf of the Trauma Audit and Research Network. Trends in head injury outcome from 1989 to 2003 and the effect of neurosurgical care: an observational study. Lancet; 366:1538-1544.

Parcell, D.L., Ponsford, J.L., Rajaratnam, S.M., Redman, J.R. (2006). Self- reported changes to nighttime sleep after traumatic brain injury. ArchPhys Med Rehabil; 87:278-285.

Ponsford, J. (2003). Sexual changes associated with traumatic brain injury. Neuropsychol Rehabil; $13: 275-289$

Sánchez, E. (2015). Salud Sexual y Calidad de vida en personas con Traumatismo Craneoencefálico Moderado. Universidad de Barcelona. Barcelona.

Sánchez, E., Honrubia, M. (2013). La utilización del cuestionario EVAS-H y EVAS_M como herramienta de valoración en las alteraciones sexuales de los pacientes con Daño Cerebral Adquirido (DCA).In: García Rojas, A.D.; Cabello Santamaría, F. Actualizaciones en sexología clínica y educativa. Huelva: Servicio. Publicaciones Universidad de Huelva; p. 253-263.

Sanders, A.M., Maestas, K.L., Pappadis, M.R., Sherer, M., Hamond, F.M., Hanks, 


\section{SALUD SEXUAL Y CALIDAD DE VIDA EN PERSONAS CON TRAUMATISMO CRANEOENCEFÁLICO MODERADO (TCEM)}

R. (2012). Sexual functioning 1 year after traumatic brain injury: findings from a prospective traumatic brain injury model systems collaborative study. Arch Phys Med Rehabil; 93:1331-1337

Shiffman, R., Shekelle, P., Overhage, M., Sfutsky. J., Grimshaw. J., Deshpande,

(2003). Standardized reporting of clinical practice guidelines: a proposal from the conference on guideline standardization. Am Inter Med; 139: 493-498.

Tagliaferri, F., Compagnone. C., Korsic, M., Servadei, F., Kraus, J. (2006). A systematic reviewof brain injury epidemiology in Europe. Acta Neurochir (Wien); 148: 255-68.

Vaquero, J. (2007). Aspectos generales de los traumatismos craneoencefálicos. Daño cerebral postraumático. Madrid: Fundación MAPFRE

Villarreal, R.G. (2012) Traumatismo Craneoencefálico, Conmoción cerebral, Escala de coma de Glasgow, Fractura craneal.[Internet] [Citado 3 de Marzo 2018].Disponible:http://neurocirugiaen-

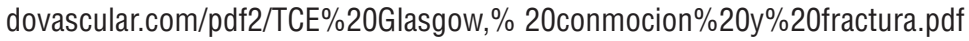

World Health Organization (2004). World reporting road traffic injury prevention. Geneva. 